\title{
INFLUÊNCIA DA POSIÇÃO SOCIAL E DO NÚMERO DE RAIOS NA ESTIMATIVA DA ÁREA DE COPA EM ARAUCÁRIA
}

\author{
Emanuel Arnoni Costa ${ }^{1}$, César Augusto Guimarães Finger ${ }^{2}$, Thiago Augusto da Cunha ${ }^{3}$ \\ ${ }^{1}$ Eng. Florestal, Mestrando em Engenharia Florestal, UFSM, Santa Maria, RS, Brasil - emanuelarnonicost@ hotmail.com \\ ${ }^{2}$ Eng. Florestal, Dr., Depto. de Ciências Florestais, UFSM, Santa Maria, RS, Brasil - cesarfinger.ufsm@ outlook.com \\ ${ }^{3}$ Eng. Florestal, Doutorando em Engenharia Florestal, UFSM, Santa Maria, RS, Brasil - dacunha.thiago@ yahoo.com
}

Recebido para publicação: 18/02/2013 - Aceito para publicação: 12/06/2013

\begin{abstract}
Resumo
O objetivo do presente estudo foi avaliar a precisão e a acurácia na estimativa da área de projeção horizontal da copa de araucária em diferentes posições sociais. Foram amostradas 294 árvores na floresta e classificadas, conforme a sua posição sociológica, em dominante, codominante e dominada. Em cada árvore foram medidos o diâmetro à altura do peito, a altura total e oito raios de copa seguindo a direção dos pontos cardeais. A área de projeção horizontal da copa foi calculada pela média dos respectivos números de raios avaliados na copa (métodos): oito, quatro e dois. As diferenças estatísticas entre os métodos foram verificadas por análise de variância com arranjo fatorial, considerando dois fatores, os métodos de área de copa e a posição social ocupada pelas árvores. Os resultados indicaram que, apesar de não haver diferença significativa entre as médias de área de projeção da copa quando medido oito e quatro raios, a análise gráfica evidenciou tendência de superestimativa da área quando calculada com quatro e dois raios em copas irregulares, frequentemente encontradas nas árvores codominantes e dominadas. Ademais, em copas simétricas, a determinação da área de projeção horizontal da copa com quatro raios pode ser empregada sem perda de acurácia.

Palavras-chave: Diâmetro de copa; superfície horizontal da copa; classe social; floresta de araucária.
\end{abstract}

\begin{abstract}
Influence of social position and number of radius in estimate of area in araucaria crown. The aim of this study was to evaluate precision and accuracy in estimating horizontal projection crown area of trees of Araucaria Angustifolia in different social positions. We sampled 294 trees in the forest and classified them according to their position in sociological position as dominant, codominant and dominated. In each tree it was measured the diameter at breast height, total height and eight crown radius following the cardinal points direction. The horizontal projection area of the crown was calculated by the average of the respective radius numbers evaluated in the crown (methods): eight, four and two. Statistical differences between among methods were verified by variance analysis with factorial arrangement, considering two factors, methods of crown area and the social position occupied by trees. The results indicated that although there was no significant difference between the mean area of crown projection when measured eight and four radius, the graphical analysis revealed tendency to overestimate the area calculated with four and two radius in irregular crown, often identified in codominant and dominated trees. Furthermore, in relation to symmetrical crowns, determining the area of horizontal projection crown with four radius can be used without loss of accuracy.

Keywords: Crown diameter; horizontal crown surface; social class; araucaria forest.
\end{abstract}

\section{INTRODUÇÃO}

Muitos modelos de árvore individual tem predito o crescimento com base em variáveis que caracterizam as dimensões da copa (COLE; LORIMER, 1994; MONSERUD; STERBA, 1996; RIVAS et al., 2005; COSTA, 2011). As dimensões atuais da copa das árvores são consequência de um efeito por ela sofrido no passado, que atua como regulador no tamanho e forma e tem implicação direta no crescimento, sugerindo a sua utilização como um descritor da concorrência.

Em árvores isoladas (livres de competição), o crescimento e dimensão da copa são máximos, podendo ser usado para o desenvolvimento de guias de manejo e na modelagem do crescimento florestal (SMITH et al., 1992; HASENAUER, 1997). 
De certa forma, atributos da copa permitem auxiliar o manejador a averiguar o potencial de árvore futuro "F" (ALBETZ; OHNEMUS, 1994). Essa compreensão também é válida quando associada a diferente posição sociológica na floresta, já que fornece informações adicionais, principalmente sobre o espaço vital adequado para seu crescimento.

A distinção das formas das árvores, dimensões e suas relações se devem, inicialmente, às pesquisas realizadas por Burger (1939), Assmann (1970), Mitscherlich (1978), Hasenauer e Monserud (1996), entre outros. No Brasil, alguns trabalhos com árvores nativas e exóticas empregaram as variáveis morfométricas na elaboração de diretrizes silviculturais para as florestas (DURLO; DENARDI, 1998; DURLO, 2001; NUTTO et al., 2001; NUTTO, 2001; TONINI; ARCO-VERDE, 2005; PADOIN; FINGER, 2010).

A Área de Projeção Horizontal da Copa (APHC) das árvores pode ser obtida de forma direta e indireta. A forma direta é possível mediante o auxílio de fotografias aéreas e imagens de satélites, entretanto, em povoamentos adensados, mistos e inequiâneos, essa técnica pode não alcançar acurácia satisfatória (CÔRTE et al., 2007; SANQUETTA et al., 2011). De forma indireta, que consiste em medir em campo os raios de copa, a atividade é trabalhosa e exige bom treinamento dos operadores, com o intuito de minimizar erros sistemáticos.

Contudo, a regularidade na forma da copa é determinante no cálculo APHC. Um aumento do número de raios de copa pode trazer maior acurácia na estimativa da área em árvores com copas assimétricas. Árvores de araucária que crescem em competição, como as codominantes e dominadas, apresentam maior irregularidade na forma da copa, advindo disso a necessidade de investigar a influência do fator posição social na quantidade de raios a serem medidos, a fim de se obterem estimativas confiáveis.

Longhi (1980) calculou a dominância de araucária utilizando a APHC pelo raio de copa médio na extensão mais longa do galho. Já outros autores computaram o diâmetro de copa variando o número de raios de copa em dois, quatro e oito raios, definidos em direções fixas, seguindo os pontos cardeais (SEITZ, 1986; WACHTEL, 1990; LISBOA, 2009; NASCIMENTO et al., 2010), ou com metodologia semelhante, mas variando os raios em diferentes posições da copa para as espécies de Araucaria angustifolia e Pinus elliottii (NUTTO, 2001; NUTTO et al., 2001), buscando obter o mais próximo da verdadeira a área de projeção de copa.

Apesar da importância, a APHC é uma variável não comumente mensurada em inventários florestais no Brasil, mas de enorme aplicação no amparo ao monitoramento das florestas (BECHTOLD, 2003; RUSSELL; WEISKITTEL, 2011; MCINTOSH et al., 2012), além de informar a zona de influência das copas (sobreposição) e o grau de concorrência da árvore (GILL et al., 2000; GETZIN et al., 2008).

Diante do exposto, o objetivo do presente estudo foi avaliar a precisão e a acurácia na estimativa da área de projeção horizontal da copa para árvores de araucária em diferentes posições sociais, considerando a medição de oito, quatro e dois raios de copa, tomados segundo os pontos cardeais.

\section{MATERIAL E MÉTODO}

\section{Área de estudo}

A floresta amostrada localiza-se numa propriedade particular com 84 hectares de floresta nativa, no município de Lages, SC $\left(27^{\circ} 48^{\prime} \mathrm{S}\right.$ e $\left.50^{\circ} 19^{\prime} \mathrm{O}\right)$. O clima da região é mesotérmico úmido (1360 a $\left.1600 \mathrm{~mm}\right)$, sem estação seca definida ( $\mathrm{Cfb}$ ), com temperatura média anual entre 13,8 e $15,8^{\circ} \mathrm{C}$ e umidade relativa de 80\%, segundo a Empresa de Pesquisa Agropecuária e Extensão Rural de Santa Catarina (EPAGRI) (2002). Os solos predominantes na região são nitossolos háplicos e cambissolos húmicos, desenvolvidos a partir de rochas basálticas, conforme Empresa Brasileira de Pesquisa Agropecuária (EMBRAPA) (1999).

\section{Levantamento dos dados}

Um total de 294 árvores de araucária foram amostradas de forma aleatória no interior da floresta. Cada árvore foi classificada quanto à posição sociológica (PS), utilizando-se o grau de exposição da copa à luz, que consistiu em: PS1 - árvore dominante: ocupava o estrato superior com alta exposição da copa à luz; PS2 - árvore codominante: ocupava o estrato intermediário, com média exposição da copa à luz; PS3 árvore dominada: ocupava o estrato inferior, com baixa exposição da copa à luz.

Em seguida, foi medido o diâmetro à altura do peito (d) com fita diamétrica, a altura total (h) com o hipsômetro Vertex IV, cujas médias se encontram na tabela 1, e oito raios de copa a partir do eixo central da árvore, ao nível do DAP, na direção dos pontos cardeais: norte (N), nordeste (NE), leste (L), sudeste (SE), sul (S), sudoeste (SO), oeste $(\mathrm{O})$ e noroeste $(\mathrm{NO})$. A bússola permitiu orientar adequadamente a direção de cada posição cardeal. Os raios de copa foram mensurados por uma única pessoa, rejeitando-se possíveis tendências de medição por outros operadores. 
Tabela 1. Resumo estatístico do diâmetro e altura total de araucária.

Table 1. Statistical summary of diameter and total height of araucária.

\begin{tabular}{lcccccc}
\hline Variável & Posição social & $\mathbf{n}$ & Mínimo & Médio & Máximo & Desvio Padrão \\
\hline \multirow{4}{*}{ Diâmetro [d] } & PS1 & 193 & 14,7 & $47,8 \mathrm{a}$ & 85,8 & 15,3 \\
& PS2 & 40 & 9,9 & $36,1 \mathrm{~b}$ & 58,9 & 12,2 \\
& PS3 & 61 & 10,2 & $28,2 \mathrm{c}$ & 49,5 & 10,5 \\
\multirow{3}{*}{ Altura [h] } & $\mathrm{T}$ & 294 & 9,9 & 42,1 & 85,8 & 16,2 \\
& PS1 & 193 & 9,1 & $18,1 \mathrm{a}$ & 24,9 & 3,3 \\
& PS2 & 40 & 7,2 & $16,1 \mathrm{~b}$ & 21,2 & 3,6 \\
\hline
\end{tabular}

d: diâmetro à altura do peito, em cm; h: altura total, em m; PS: posição sociológica: 1- dominante 2- codominante, 3- dominada; T: todo conjunto de dados desconsiderando a posição social; n: número de indivíduos; * Médias seguidas da mesma letra nas colunas não diferem estatisticamente pelo teste de Tukey-Kramer com significância de 5\% ( $\operatorname{Pr}>0,05)$.

\section{Avaliação dos métodos}

Os métodos de APHC foram avaliados pela expressão 1, utilizando-se a média aritmética do número de raios e definida como: APHC8- raios de copa medido nos pontos cardeais (N, NE, L, SE, S, SO, $\mathrm{O}$ e NO); APHC4- raios de copa medidos nos pontos cardeais (N, L, S e O); e APHC2- raios de copa medidos no ponto cardeal de maior raio de copa e de seu prolongamento a $180^{\circ}$.

$$
\mathrm{APHC}_{\mathrm{i}}=\pi \cdot \mathrm{rc}_{\mathrm{i}}^{2}
$$

Em que: $\mathrm{rc}_{\mathrm{i}}=$ raio de copa da $i$-ésima árvore, $\mathrm{em} \mathrm{m}$.

O erro de amostragem relativo obtido para os métodos de determinação da APHC foi calculado para população infinita, conforme a expressão encontrada em Péllico Netto e Brena (1997).

Para verificar o efeito dos métodos adotados na estimação da APHC, utilizou-se um modelo linear de ANOVA por mínimos quadrados ordinários com dois fatores: número de raios empregados no cálculo e a posição social ocupada pelas árvores, formando nove tratamentos $(\mathrm{T})$ : T1 = APHC8*PS1; $\mathrm{T} 2=\mathrm{APHC} 8 * \mathrm{PS} 2 ; \mathrm{T} 3=\mathrm{APHC} 8 * \mathrm{PS} 3 ; \mathrm{T} 4=\mathrm{APHC} 4 * \mathrm{PS} 1 ; \mathrm{T} 5=\mathrm{APHC} 4 * \mathrm{PS} 2 ; \mathrm{T} 6=\mathrm{APHC} 4 * \mathrm{PS} 3 ;$ $\mathrm{T} 7=\mathrm{APHC} 2 * \mathrm{PS} 1 ; \mathrm{T} 8=\mathrm{APHC} 2 * \mathrm{PS} 2 ; \mathrm{T} 9=\mathrm{APHC} 2 * \mathrm{PS} 3$. A área calculada com APHC 8 foi considerada como sendo a mais próxima da verdadeira APHC, sendo a testemunha das análises estatísticas.

Com o intuito de cumprir com os supostos dos mínimos quadrados ordinários, os dados da variável dependente $\left(\mathrm{APHC}_{\mathrm{i}}\right)$ foram transformados pelo método Box e Cox (BOX; COX, 1964). O experimento foi desbalanceado com diferente número de unidades amostrais (árvore) nos nove tratamentos.

Em seguida, considerou-se como verdadeira (ou mais próximo do real) a APHC calculada por oito raios. Assim, foi possível medir o erro cometido quando usado quatro (APHC4) e dois raios (APHC2) para cada posição social, utilizando as expressões 2 e 3.

$$
\begin{aligned}
& \varepsilon^{(\mathrm{APHC} 4)}=\mathrm{APHC} 4-\mathrm{APHC} 8 \\
& \varepsilon^{(\mathrm{APHC} 2)}=\mathrm{APHC} 2-\mathrm{APHC} 8
\end{aligned}
$$

Para obtenção do viés geral e da variabilidade dos métodos de APHC, o erro médio (expressão 4) e o desvio padrão (expressão 5) foram calculados para os valores da expressão 2 e 3.

$$
\begin{aligned}
& \bar{\varepsilon}(.)^{*}=\frac{1}{\mathrm{n}} \sum_{\mathrm{i}=1}^{\mathrm{n}} \varepsilon(.)_{\mathrm{i}} \\
& \mathrm{sd}=\sqrt{\frac{1}{\mathrm{n}-1} \sum_{\mathrm{i}=1}^{\mathrm{n}}\left(\varepsilon(.)_{\mathrm{i}}-\bar{\varepsilon}(.)^{*}\right)^{2}}
\end{aligned}
$$

Em que: $\bar{\varepsilon}=$ erro médio;

sd $=$ desvio padrão;

$(.)^{*}=\mathrm{APHC} 4$ e APHC2;

$\varepsilon(.)_{\mathrm{i}}=\varepsilon^{(\mathrm{APHC} 4)} \mathrm{e}^{\varepsilon^{(\mathrm{APHC} 2)}}$, obtida por posição social. 
Ademais, para medir a relação entre as APHC obtidas por diferentes números de raios de copa, utilizou-se o modelo linear 6.

$$
y=\beta_{0}+\beta_{1} x+\varepsilon
$$

Em que: $\mathrm{x}=\mathrm{APHC}$, assumido como a área verdadeira;

y = área obtida com menor número de raios de copa APHC4 e APHC2, respectivamente.

Dessa forma, na equação 6 assume-se que caso o número de raios não tenha influência, espera-se um intercepto igual a zero e um coeficiente angular igual a 1 . O processamento dos dados foi realizado no sistema de análise estatística SAS V. 9.1 (SAS Institute Inc., 2004).

\section{RESULTADOS E DISCUSSÕES}

A amostra de 294 árvores representou bem as classes de posição sociológica em toda a amplitude de distribuição diamétrica, recobrindo diâmetros entre 9,9 e $85,8 \mathrm{~cm}$. O diâmetro e a altura média entre as posições sociais exibiram diferenças estatísticas significantes, confirmando que cada grupo sociológico possui dimensões distintas (Tabela 1).

O resumo estatístico dos métodos de área de copa avaliados por posição social mostrou, em média, um aumento da superfície de projeção da copa, quando ocorre redução do número de raios empregados no cálculo, e, consequentemente, o aumento do erro quando comparado com a área calculada com oito raios (Tabela 2). Entretanto, o teste de média de Tukey-Kramer realizado para todo o conjunto de dados não confirmou diferença significante entre métodos com medidas em quatro e oito raios.

Tabela 2. Resumo estatístico dos métodos de APHC por posição social em araucária.

Table 2. Summary of statistical methods APHC for social position in araucária.

\begin{tabular}{lcccccc}
\hline Métodos & Posição Social & Mínimo & Média & Máximo & CV\% & Ear (\%) \\
\hline APHC8 & PS1 & 10,4 & 105,4 & 392,1 & 63,8 & 7,60 \\
APHC4 & $(\mathrm{n}=193)$ & 9,6 & 103,7 & 306,4 & 64,3 & 7,65 \\
APHC2 & & 11,5 & 123,8 & 487,7 & 64,8 & 7,71 \\
\hline APHC8 & PS2 & 7,2 & 56,3 & 141,2 & 60,9 & 16,21 \\
APHC4 & $(\mathrm{n}=40)$ & 4,9 & 60,3 & 159,3 & 68,5 & 18,23 \\
APHC2 & & 8,2 & 70,8 & 257,0 & 73,8 & 19,65 \\
\hline APHC8 & PS3 & 0,9 & 46,0 & 132,0 & 74,5 & 15,94 \\
APHC4 & $(\mathrm{n}=61)$ & 3,7 & 47,1 & 145,8 & 74,9 & 16,01 \\
APHC2 & & 5,3 & 60,6 & 236,2 & 79,8 & 17,06 \\
\hline APHC8 & $\mathrm{T}$ & 0,9 & $86,2 \mathrm{a}$ & 392,1 & 73,9 & 8,48 \\
APHC4 & $(\mathrm{n}=294)$ & 3,7 & $86,0 \mathrm{a}$ & 306,4 & 73,6 & 8,45 \\
APHC2 & & 5,3 & $103,5 \mathrm{~b}$ & 487,7 & 74,0 & 8,49 \\
\hline APHC1
\end{tabular}

APHCi: área de projeção horizontal da copa considerando os respectivos números de raios de copa usados no cálculo, em $\mathrm{m}^{2}$ PS: posição sociológica: 1-dominante, 2-codominante, 3-dominada; T: todo conjunto de dados desconsiderando a posição social; n: número de indivíduos; CV\%: coeficiente de variação; Ear: erro de amostragem relativo; * Médias seguidas da mesma letra nas colunas não diferem estatisticamente pelo teste de Tukey-Kramer com significância de 5\% (Pr>0,05).

As estimativas do coeficiente de variação foram homogêneas para as árvores de posição social dominante. Na posição social codominante e dominada, o valor do coeficiente de variação tendeu a aumentar à medida que se reduziu o número de raios de copa no cálculo da área (Tabela 2). A quantidade de variação encontrada na posição social codominante foi consequência dos diferentes graus de competição e luminosidade a que essas copas estavam submetidas na floresta.

Em árvores dominantes, o erro de amostragem relativo foi menor que $8 \%$, com tendência a diminuir à medida que aumenta o número de raios de copa utilizados no cálculo. Para o grupo das árvores codominantes e dominadas, o erro de amostragem relativo variou de $15,0 \%$ a $20,0 \%$, aproximadamente. Por outro lado, a variação total para os métodos analisados tendeu a permanecer alta, com valor próximo a $74 \%$.

A tendência linear média das APHC relacionada com o aumento do diâmetro (representado pela área basal individual ou área transversal) das árvores dentro da posição social alcançou ajustes entre $\mathrm{R}^{2}=54,0 \% \mathrm{e}$ 
$\mathrm{R}^{2}=79,0 \%$, conforme mostrados na figura 1 . Todas as equações exibiram os coeficientes $\hat{\beta}_{0}$ e $\hat{\beta}_{1}$ significantes a 5\% de probabilidade. Os valores dos coeficientes de determinação obtidos com as regressões ajustadas apresentou conformidade com outros estudos para a espécie, em que autores utilizaram métodos distintos ao do presente trabalho para a determinação da APHC, com valor de $\mathrm{R}^{2}=78,0 \%$ (SANQUETTA et al., 2011).
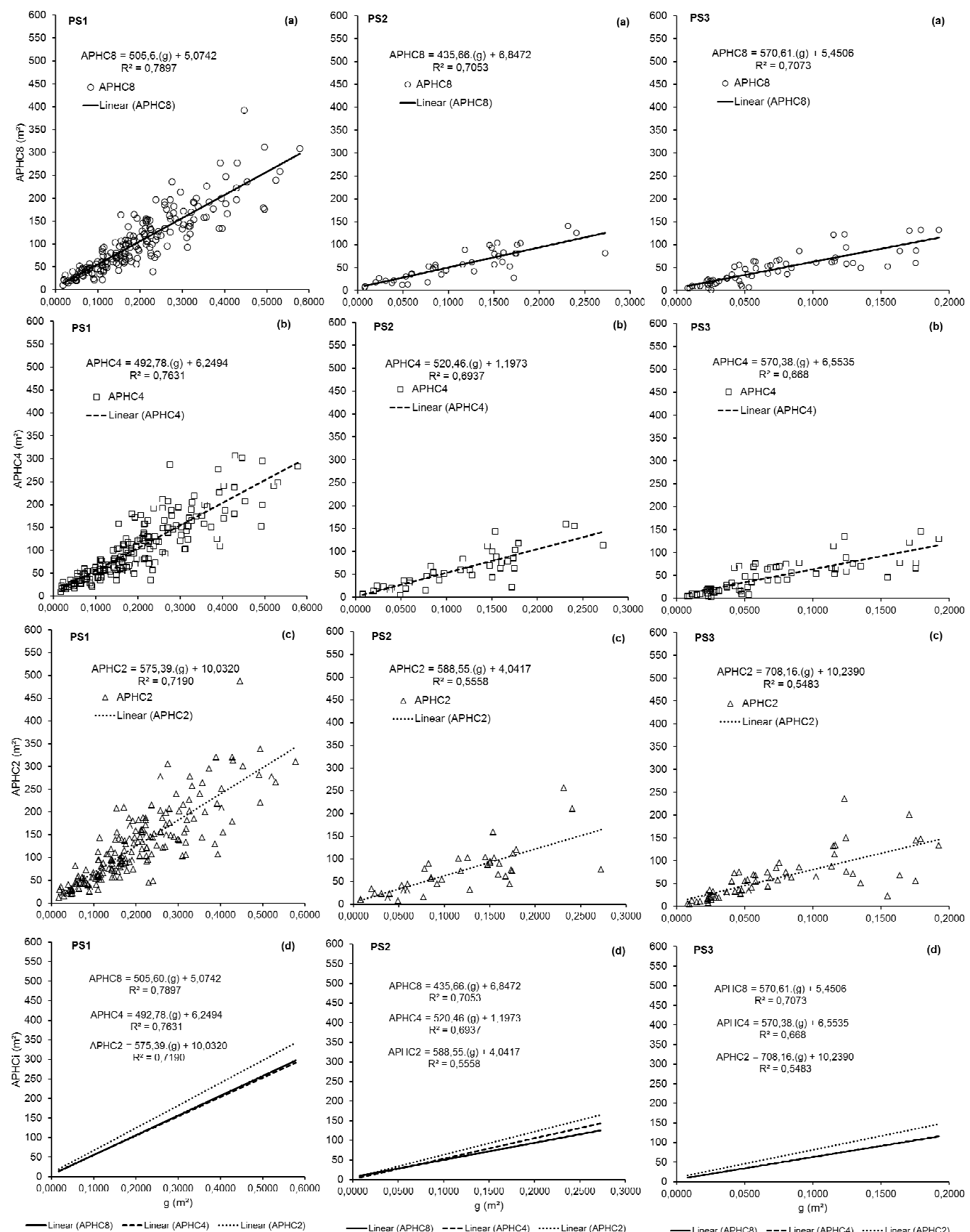

Figura 1. APHC observada e estimada (regressão linear) utilizando oito raios (a), quatro raios (b) e dois raios (c), para a posição social dominante (PS1), codominante (PS2) e dominada (PS3).

Figure 1. APHC observed and estimated (linear regression) using eight rays (a), four rays (b), and two rays (c) for the social position dominant (PS1), codominant (PS2) and dominated (PS3). 
Lockhart et al. (2005) recomendaram o uso de imagens digitais para a determinação do raio de copa, em seguida prognosticado pelo diâmetro, com o estudo desenvolvido em seis espécies. Aplicando modelos lineares simples, obtiveram ajustes com $\mathrm{R}^{2}$ variando de 56,0 a $85,0 \%$. No entanto, para descrever a mesma relação do presente estudo (área de copa e área basal individual) em Pinus elliotti, os ajustes foram próximos a $\mathrm{R}^{2}=47,0 \%$, usando o método de quatro raios medidos em campo em orientação cardeal fixa (N, S, L e O) (NUTTO et al., 2001).

Os estudos publicados que descreveram a relação do diâmetro de copa com o diâmetro à altura do peito para araucária referiram tendência linear ou levemente curvilinear entre essas variáveis, sem diferenciar classes sociológicas (LONGHI, 1980; OLIVEIRA, 1982; SEITZ, 1991; NASCIMENTO et al., 2010). No entanto, vale destacar que a tendência linear simples pode se tornar quadrática com o aumento do porte das árvores (diâmetro à altura do peito superior a $90 \mathrm{~cm}$, aproximadamente), havendo, pois, a necessidade de adição de um termo quadrático como variável independente no modelo, como pode ser observado em Nutto (2001) e Lisboa (2009), indicando que, a partir dessa dimensão, as árvores continuam crescendo em diâmetro à altura do peito, mas tendem a estabilizar o seu crescimento em diâmetro de copa.

Na figura 1, a variação na APHC, atribuída à quantidade de raios utilizada para calculá-la, foi maior nas posições sociais codominante (PS2) e dominada (PS3). De fato, durante a medição em campo, houve maior dificuldade para a quantificação dos limites da copa das árvores nessas posições. Em árvores dominantes, a irregularidade nos raios de copa foi menor, formando com isso copas com projeção horizontal mais circular. Essas árvores, frequentemente, encontram-se sem competidores laterais, possibilitando a formação de galhos com comprimentos mais regulares em todas as direções.

$\mathrm{Na}$ análise de variância para os tratamentos, constatou-se que a condição de homogeneidade de variância da variável dependente (APHC) não foi atendida, conforme o resultado do teste de Bartlett $\left(\mathrm{X}^{2}=128,5\right.$; Prob. $\left.<0,0001\right)$. A transformação Box e Cox pela potência 0,267 $\left(\mathrm{APHC}^{0,267}\right)$ permitiu obter variâncias homogêneas Bartlett $\left(X^{2}=2,5\right.$; Prob. $\left.<0,9623\right)$ e atender ainda a condição de normalidade dos dados, conforme o teste de Cramer Von Mises (W-Sq = 0,12553; Prob. $>\mathrm{D}=0,0509$ ).

Os dados transformados APHC passaram a apresentar distribuição normal, permitindo analisar a variância para os tratamentos, cujo resultado expôs diferença significante entre si (Prob. <0,0001). O contraste das médias dos tratamentos mostrou o efeito do método de cálculo da área de projeção copa em uma mesma posição social, bem como entre as posições sociais (Tabela 3).

Tabela 3. Teste de t das médias APHC8, APHC4 e APHC2 por posição social em araucária.

Table 3. T test averages APHC8, APHC4 and APHC2 by social position in araucária.

\begin{tabular}{cccccccccc}
\hline \multirow{2}{*}{ i/j } & \multicolumn{9}{c}{ Tratamentos } \\
\cline { 2 - 22 } & T1 & T2 & T3 & T4 & T5 & T6 & T7 & T8 & T9 \\
\hline T1 & & $4,79^{*}$ & $7,82^{*}$ & $0,24^{\text {ns }}$ & $4,60^{*}$ & $7,74^{*}$ & $-2,32^{*}$ & $3,38^{*}$ & $5,64^{*}$ \\
T2 & $<0,0001$ & & $1,61^{\text {ns }}$ & $-4,64^{*}$ & $-0,15^{\text {ns }}$ & $1,50^{\text {ns }}$ & $-6,14^{*}$ & $-1,10^{\text {ns }}$ & $-0,01^{\text {ns }}$ \\
T3 & $<0,0001$ & 0,1069 & & $-7,73^{*}$ & $-1,77^{\text {ns }}$ & $-0,13^{\text {ns }}$ & $-9,50^{*}$ & $-2,82^{*}$ & $-1,83^{\text {ns }}$ \\
T4 & 0,8086 & 0,0001 & $<0,0001$ & & $4,45^{*}$ & $7,57^{*}$ & $-2,56^{*}$ & $3,23^{*}$ & $5,47^{*}$ \\
T5 & $<0,0001$ & 0,8838 & 0,0763 & $<0,0001$ & & $1,66^{\text {ns }}$ & $-5,95^{*}$ & $-0,95^{\text {ns }}$ & $0,15^{\text {ns }}$ \\
T6 & $<0,0001$ & 0,1340 & 0,8980 & $<0,0001$ & 0,0972 & & $-9,34^{*}$ & $-2,70^{*}$ & $-1,70^{\text {ns }}$ \\
T7 & 0,0207 & $<0,0001$ & $<0,0001$ & 0,0107 & $<0,0001$ & $<0,0001$ & & $4,73^{*}$ & $7,25^{*}$ \\
T8 & 0,0008 & 0,2738 & 0,0049 & 0,0013 & 0,3429 & 0,0070 & $<0,0001$ & & $1,19^{\text {ns }}$ \\
T9 & 0,0001 & 0,9888 & 0,0677 & $<0,0001$ & 0,8835 & 0,0893 & $<0,0001$ & 0,2345 & \\
\hline
\end{tabular}

ns: não significante; *: significante em Pr. <0,05. Comparações múltiplas ajustadas por Tukey-Kramer. A diagonal superior mostra os valores de t e a inferior as probabilidades. T1: PS1*APHC8; T2: PS2*APHC8; T3: PS3*APHC8; T4: PS1*APHC4; T5: PS2*APHC4; T6: PS3*APHC4; T7: PS1*APHC2; T8: PS2*APHC2; T9: PS3*APHC2.

A comparação dos métodos APHC8 e APHC4 para árvores dominantes apontou não haver diferença significante entre os dois métodos T4-T1 (Prob. <0,8086), e para árvores codominantes T5-T2 (Prob. <0,8838) e dominadas T6-T3 (Prob. <0,8980) (Tabela 3). A interpretação do resultado com base no valor médio indicou ser possível calcular a área da copa a partir da média de quatro ou oito raios sem perda de acurácia. 
Por outro lado, a análise da distribuição de valores na figura 1d, referentes à posição social de árvores codominantes (PS2), não confirmou esse resultado, pois a linha de regressão do método com quatro raios superestimou áreas de projeção de copa.

Quando o método APHC8 foi comparado com APHC2 em árvores dominantes, esta diferença foi significante em T7-T1 (Prob. <0,0207), com os valores de área de copa tendendo a superestimativa quando calculado com base em medidas tomadas sob dois raios de copa (Figura 1d - PS1).

O método de APHC4 comparado com APHC2 para árvores dominantes indicou diferença significante para os tratamentos T7-T4 (Prob. <0,0107). Ao contrário, em árvores codominantes e dominadas, os valores analisados das áreas médias não foram relevantes (Prob. <0,3429) para os tratamentos T8-T5 e (Prob. <0,0893) para T9-T6, respectivamente. O resultado deve ser tomado com cuidado, pois refletiu a dimensão da média da distribuição dos dados, não evidenciando a tendência de superestimava das áreas em relação às calculadas com oito raios. A mesma observação deve ser considerada para as áreas de projeção horizontal calculadas com dois raios para árvores codominantes e dominada, pois os resultados apresentaram superestimava nas árvores de maior diâmetro, conforme evidenciado na figura $1 \mathrm{~d}$.

Nutto et al. (2001), utilizando diferente procedimento na análise de métodos de determinação APHC para espécies de Pinus elliotti, concluíram que o método de oito raios de copa com ângulos variáveis foi mais preciso que o método de quatro raios de copa com ângulos fixos.

Grote (2003) descreveu métodos para a estimativa dos raios de copa em diferentes direções cardeais usando como variáveis independentes dos modelos o diâmetro da árvore, a altura e o comprimento da copa, para as espécies de Picea abies L. Karst e Fagus sylvatica L. em povoamentos puros e mistos dessas espécies. Gill et al. (2000), avaliando a modelagem dos raios de copa em coníferas, testaram as variáveis diâmetro, altura, altura na base da copa, classe de copa, área basal e número de árvores por hectare como sendo variáveis independentes dos modelos, confirmando que o diâmetro seria a variável independente mais adequada para descrever essa relação.

Fiala et al. (2006), comparando cinco métodos para a estimativa da superfície de copa, relataram que as medidas da copa feitas de forma indireta no campo devem ser recomendadas em objetivos específicos, principalmente quando a precisão torna-se um objetivo importante.

$\mathrm{O}$ viés encontrado com os métodos APHC4 e APHC2 em relação ao APHC8, admitindo que o último assemelha-se às dimensões mais próximas da verdadeira área de copa, pode ser visualizado na tabela 4. Os valores encontrados mostraram que ocorre um aumento da dispersão dos erros, com tendência de superestimar áreas de copa quando usado o método APHC2 (Figura 2). Essas diferenças foram mais expressivas em árvores de posição social dominada e principalmente nas codominantes, em que o erro médio alcançou $14,54 \mathrm{~m}^{2}$ e o desvio padrão foi de $86,61 \mathrm{~m}^{2}$, conforme a tabela 4 .
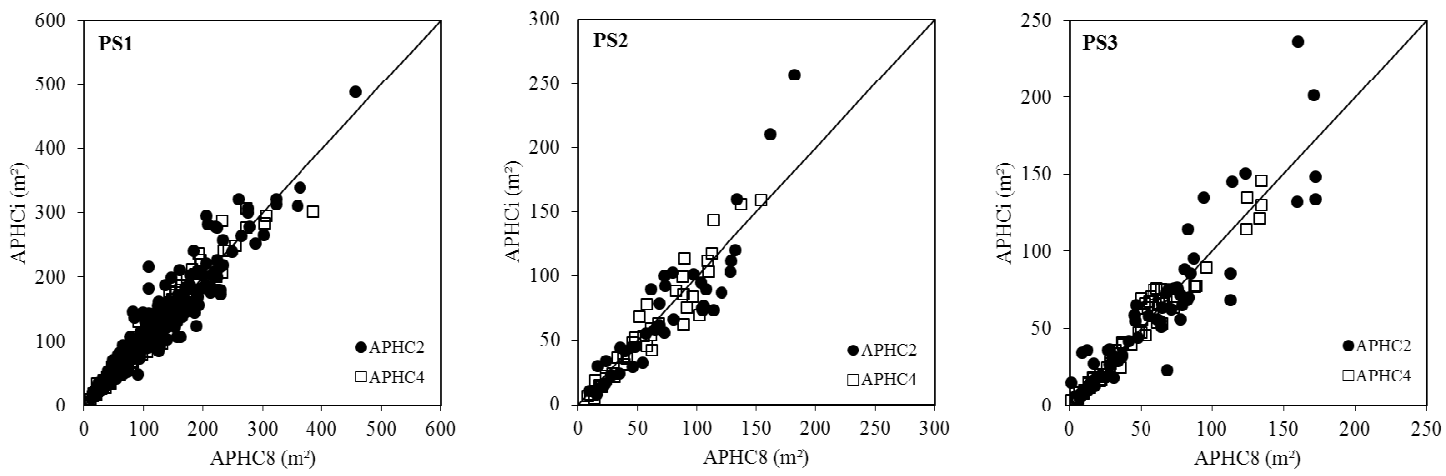

Figura 2. Dispersão linear dos erros usando o método APHC2 e APHC4 em relação ao APHC8, em posição social dominante (PS1), codominante (PS2) e dominada (PS3).

Figure 2. Linear dispersion of errors using the method APHC2 and APHC4 compared to APHC8 in social position dominant (PS1), codominant (PS2) and dominated (PS3). 
Tabela 4. Determinação do viés obtido para os métodos APHC entre as posições sociais.

Table 4. Determination of bias obtained for methods APHC between social positions.

\begin{tabular}{lccc}
\hline Posição sociológica & APHC $_{\mathbf{i}}$ & $\bar{\varepsilon}$ & sd \\
\hline \multirow{2}{*}{ PS1 } & 4 & $-1,36$ & 14,17 \\
& 2 & 18,75 & 27,12 \\
PS2 & 4 & 3,97 & 45,60 \\
& 2 & 14,54 & 86,61 \\
PS3 & 4 & 1,09 & 7,28 \\
& 2 & 14,56 & 21,91 \\
\hline APHCi: área de projeção horizontal da copa considerando os respectivos números de raios de \\
copa usados no cálculo; PS: posição sociológica: 1: dominante, 2: codominante, 3: dominada; \\
$\bar{\varepsilon}=$ erro médio, em m $^{2} ;$ sd = desvio padrão, em m ${ }^{2}$.
\end{tabular}

\section{CONCLUSÕES}

De acordo com a análise e discussão dos resultados, chegou-se às seguintes conclusões:

- A área de projeção horizontal da copa calculada com oito raios teve maior acurácia, descrevendo melhor a verdadeira superfície da copa. A comparação estatística das áreas médias da copa não indentifica diferenças significantes quando usados oito ou quatro raios; porém a análise gráfica demonstra a clara tendência de superestimativa das áreas quando calculada com quatro e dois raios em copa de forma irregular, frequente nas árvores codominantes e dominadas.

- Árvores codominantes de araucária devem ter a área de projeção horizontal da copa calculada por oito raios, sendo o fator de maior influência a regularidade da copa nos quadrantes.

- A determinação da área de projeção da copa com a medição de quatro raios pode ser empregada em copas simétricas, não havendo perda de acurácia.

- O custo adicional necessário para a medição do maior número de raios na copa não foram considerados neste estudo, no entanto deve-se equilibrar a precisão exigida com maior necessidade de tempo e recurso financeiro.

\section{REFERÊNCIAS}

ALBETZ, P.; OHNEMUS, K. Der Z-Baum-Bestockungsgrad (Definition, Herleitung, Anwendung), Allgemeine Forst und Jagdzeitung, Frankfurt am Main, 165. Jg., 10-12: 177 - 185, 1994.

ASSMANN, E. The principles of forest yield study. Oxford: Pergamon Press, 1970. 506 p.

BECHTOLD, W. A. Crown-diameter prediction models for 87 species of stand-grown trees in the eastern United States. Southern Journal of Applied Forestry, Washington, v. 27, p. 269 - 278, 2003.

BOX, G. E. P.; COX, D. R. An analysis of transformations. Journal of the Royal Statistical Society, London, B-26, p. 211 - 243. 1964.

BURGER, H. B. Zuwachs in zwei hiebsreifen Fichtenbeständen. Mitteilungen der Schweizerischen Anstalt für das Forstliche Versuchswesen, v. 21, p. 147 - 176, 1939.

COLE, W. G.; LORIMER C. G. Predicting tree growth from crown variables in managed Northern hardwood stands, Forest Ecology and Management, Amsterdam, v. 67, p. 159 - 175, 1994.

CÔRTE, A. P. D.; CANALEZ, G. G.; SANQUETTA, C. R. Caracterização da estrutura vertical e do dossel de um fragmento de Floresta Ombrófila Mista. Irati. Ambiência, v. 3, p. 13 - 25, 2007.

COSTA, E. A. Influência de variáveis dendrométricas e morfométricas da copa no incremento periódico de Araucaria angustifolia (Bertol.) Kuntze, Lages, SC. 140 f. Dissertação (Mestrado em Engenharia Florestal), Universidade Federal de Santa Maria, Santa Maria. 2011.

DURLO, M. A. Relações morfométricas para Cabralea canjerana (Well.) Mart. Ciência Florestal, Santa Maria, v. 11, n. 1, p. 141 - 150, 2001. 
DURLO, M. A.; DENARDI, L. Morfometria de Cabralea canjerana, em mata secundária nativa do Rio Grande do Sul. Ciência Florestal, Santa Maria, v. 8, n. 1, p. 55 - 66, 1998.

EMPRESA BRASILEIRA DE PESQUISA AGROPECUÁRIA (EMBRAPA). Sistema brasileiro de classificação de solos. Brasília: Embrapa Produção de Informação; Rio de Janeiro: Embrapa Solos, 1999. $412 \mathrm{p}$.

EMPRESA DE PESQUISA AGROPECUÁRIA E EXTENSÃO RURAL DE SANTA CATARINA (EPAGRI). Dados e Informações Biofísicas da Unidade de Planejamento Regional Planalto Sul Catarinense - UPR 3, Florianópolis, 2002. 70 p.

FIALA, A. C. S.; GARMAN, S. L; GRAY, A. Comparison of five canopy-cover estimation techniques in the western Oregon Cascades. Forest Ecology and Management, Amsterdam, v. 232, p. 188 - 197, 2006.

GROTE, R. Estimation of crown radii and crown projection area from stem size and tree position. Annals of Forest Science, Les Ulis, v. 60, p. 393 - 402, 2003.

GETZIN, S.; WIEGAND, K.; SCHUMACHER, J.; GOUGEON, F. A. Scale-dependent competition at the stand level assessed from crown areas. Forest Ecology and Management, Amsterdam, v. 255, p. 2478 2485, 2008.

GILL, S. J.; BIGING, G. S.; MURPHY, E. C. Modeling conifer tree crown radius and estimating canopy cover. Forest Ecology and Manegement, Amsterdam, v. 126, p. 405 - 416, 2000.

HASENAUER, H. Dimensional relationships of open-grown trees in Austria. Forest Ecology and Manegement, Amsterdam, v. 96, p. 197 - 206, 1997.

HASENAUER, H.; MONSERUD, R. A. A crown ratio model for Austrian forests. Forest Ecology and Manegement, Amsterdam, v. 84, p. 49 - 60, 1996.

LISBOA, G. S. Fotografias aéreas de escala grande e imagem IKONOS-2 no mapeamento de copa de Araucaria angustifolia. 104 p. Dissertação (Mestrado em Engenharia Florestal), UNICENTRO, Irati, 2009.

LOCKHART, B. R.; WEIH, R. C.; SMITH, J. K. M. Crown radius and diameter at breast height relationships for six bottomland hardwood species. Journal of the Arkansas Academy of Science, v. 59, p. $110-115,2005$.

LONGHI, S. J. A estrutura de uma floresta natural de Araucaria angustifolia (Bert.) O. Ktze, no Sul do Brasil. 198 p. Dissertação (Mestrado em Engenharia Florestal) - Universidade Federal do Paraná, Curitiba, 1980.

MCINTOSH, A. C. S.; GRAY, A. N.; GARMAN, S. L. Estimating canopy cover from standard Forest Inventory Measurements in Western Oregon. Forest Science, Bethesda, v. 58, p. 154 - 167, 2012.

MITSCHERLICH, G. Wald, Wachstum und Umwelt. Eine Einführung in die ökologischen Grundlagen des Waldwachstums. Erster Band: Form und Wachstum von Baum und Bestand. II Auflage. Frankfurt am Main: J, D. Sauerländer's Verlag, 1978. 144 p.

MONSERUD, R.; STERBA, H. A. A basal area increment model for individual trees growing in even-anduneven-aged forest stands in Austria. Forest Ecology and Management, Amsterdam, v. 80, p. 57 - 80, 1996.

NASCIMENTO, R. G. M.; MACHADO, S. A.; FIGUEIREDO, D. J.; AUGUSTYNCZIK, A. L. D.; CAVALHEIRO, R. Relações dendrométricas de Araucaria angustifolia. Nota científica, Colombo, v. 30, n. 64, p. $369-374,2010$.

NUTTO, L.; TONINI, H.; BORSOI, G. A.; MOSKOVICH, F. A.; SPATHEF, P. Utilização dos parâmetros da copa para avaliar o espaço vital em povoamentos de Pinus elliottii Engelm. Boletim de Pesquisa Florestal, v. 42, p. 110 - 122, 2001.

NUTTO, L. Manejo do crescimento diamétrico de Araucaria angustifolia (Bert.) O. Kuntze baseado na árvore individual. Ciência Florestal, Santa Maria, v. 11, n. 2, p. 9 - 25, 2001. 
OLIVEIRA, Y. M. M. Características entre parâmetros dendrométricos em Araucaria angustifolia (Bert.) O. Kuntze utilizando fotografias aéreas. Colombo, Boletim de Pesquisa Florestal, n. 5, p. 69 - 105, 1982.

PADOIN, V.; FINGER, C. A. G. Relações entre as dimensões da copa e a altura das árvores dominantes em povoamentos de Pinus taeda L. Ciência Florestal, Santa Maria, v. 20, n. 1, p. 95 - 105, 2010.

PÉLLICO NETTO, S.; BRENA, D. A. Inventário florestal. Curitiba: Edição do autor, 1997. 316 p.

RIVAS, J. J. C.; ÁLVAREZ, J. G.; AGUIRRE, G. O.; HERNÁNDEZ, F. J. The effect of competition on individual tree basal area growth in mature stands of Pinus cooperi Blanco in Durango (Mexico). European Journal of Forest Research, Georgetown, v. 124, p. 133 - 142, 2005.

RUSSELL, M. B.; WEISKITTEL, A. R. Maximum and largest crown width equations for 15 tree species in Maine. Northern Journal of Applied Forestry, Bethesda, v. 28, p. 84 - 91, 2011.

SANQUETTA, C. R.; CÔRTE, A. P. D.; JACON, A. D. Crown area and trunk diameter relationship for tree species at a mixed-araucaria natural forest in the mid-southern Parana state, Brazil. Floresta, Curitiba, PR, v. 41, n. 1, p. 63 - 72, 2011.

SAS Institute. The SAS System for Windows. Cary: SAS Institute. 2004.

SEITZ, R. A. Erste Hinweise für die waldbauliche Behandlung von Araukarienwäldern. Annales des Sciences Forestiéres, n. 43, p. 327 - 338, 1986.

Avanços na silvicultura de Pinus sp. e Araucaria angustifolia no Sul do Brasil. In: SIMPÓSIO INTERNACIONAL “O DESAFIO DAS FLORESTAS NEOTROPICAIS”, 1991, Curitiba. O desafio das florestas neotropicais. Curitiba: Universidade Federal do Paraná; Freiburg: Universidade Albert Ludwig, p. 153 - 176. 1991.

SMITH, W. R.; FARRAR JR., R. M.; MURPHY, P. A.; YEISER, J. L.; MELDAHL, R. S.; KUSH, J. S. Crown and basal area relationships of open-grown southern pines for modeling competition and growth. Canadian Journal of Forest Research, Ottawa, v. 22, p. 341 - 347, 1992.

TONINI, H.; ARCO-VERDE, M. F. Morfologia da copa para avaliar o espaço vital de quatro espécies nativas da Amazônia. Pesquisa Agropecuária Brasileira, Brasília, v. 40, n. 7, p. 633 - 638, 2005.

WACHTEL, G. Untersuchungen zu Struktur und Dynamik eines Araukarien-Naturwaldes in Südbrasilien. 180 p. Dissertação (Doutorado) - Universität Freiburg, Freiburg, 1990. 